\title{
Clopidogrel Load Reduces Emboli in Carotid Artery Stenosis With Free-Floating Thrombus
}

\author{
Brett R. Graham, Bijoy K. Menon
}

Key words: Stroke imaging, stroke, carotid artery disease, transcranial Doppler

doi:10.1017/cjn.2017.48

Can J Neurol Sci. 2017; 44: 594-596

A 62-year-old male presented subacutely to a tertiary medical centre after experiencing sudden-onset dysarthria and right arm weakness. The entirety of the symptoms lasted 30 minutes before partially resolving, leaving the man with a minimally weak and clumsy right hand. Initial noncontrast computed tomography (CT) of the head showed no acute findings and CT angiogram (CTA) showed a severely stenosed $(80 \%)$ proximal left internal carotid artery (ICA) with an associated free-floating thrombus (FFT), which extended $15 \mathrm{~mm}$ caudally from its attachment to the posterior aspect of the proximal ICA (Figure 1). The patient was started on intravenous unfractionated heparin and aspirin and was admitted to the stroke service for further observation and workup.

Magnetic resonance imaging of the brain showed multiple acute infarcts scattered throughout the left middle cerebral artery vascular territory, suggestive of an atheroembolic shower.
On the third day, the patient experienced fluctuating neurologic symptoms consisting of dysarthria and dense right arm hemiparesis, despite being normotensive and having a therapeutic activated partial thromboplastin time. Repeat noncontrast computed tomography showed no new acute features, and CTA showed no large vessel occlusion or change in the FFT. The symptoms resolved shortly thereafter. A similar event occurred on day 4 and a transcranial Doppler (TCD) was placed for emboli detection. Over a course of 1 hour, 84 high-intensity transient signals (HITS) were recorded. An oral load of clopidogrel $600 \mathrm{mg}$ was given to the patient and aspirin was discontinued. TCD for emboli detection 4 hours later showed no further HITS over a 1-hour recording period (Figure 2). Repeat emboli detection the next day again showed no HITS over a 1-hour period. No fluctuations in neurological status were noted after the clopidogrel load. Repeat CTA performed the following day showed partial resolution of the FFT and ICA stenosis.
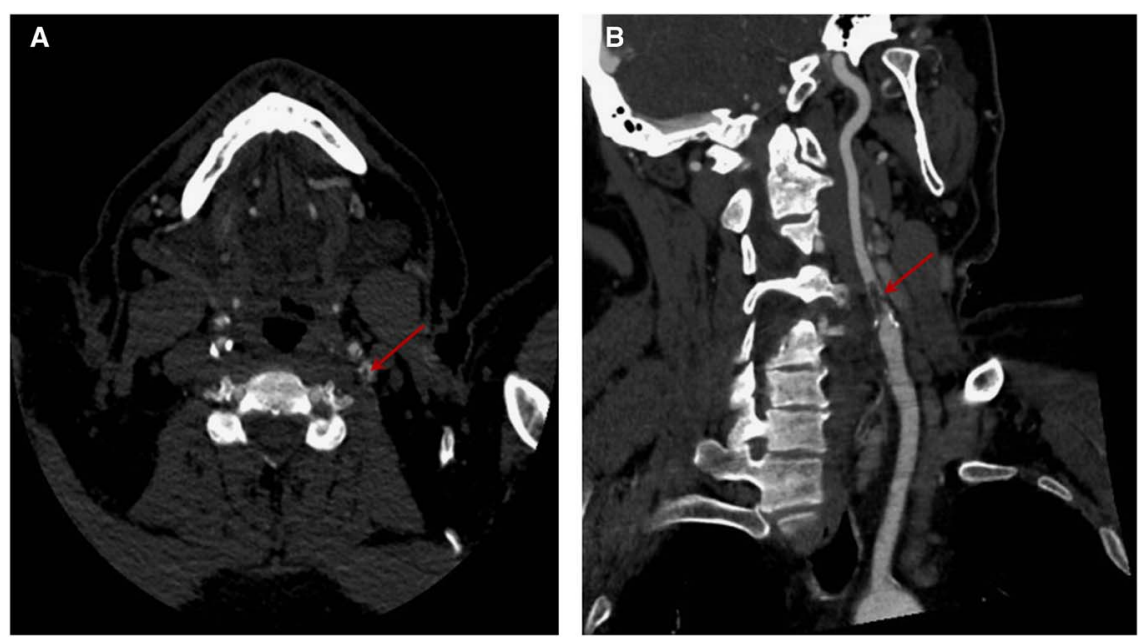

Figure 1: Free-floating thrombus (FFT) visualized by CTA in the left ICA in the axial (A) and sagittal (B) plains. Circumferential blood flow and the elongated nature of the thrombus are depicted in $A$ and $B$, respectively.

From the Department of Neuroscience (BRG), Calgary Stroke Program, Cumming School of Medicine, University of Calgary, Calgary, AB, Canada; Departments of Clinical

Neurosciences, Radiology, and Community Health Sciences (BKM), Cumming School of Medicine, University of Calgary, Calgary, AB, Canada.

Received November 18, 2016. Final Revisions Submitted February 3, 2017. Date of Acceptance March 4, 2017.

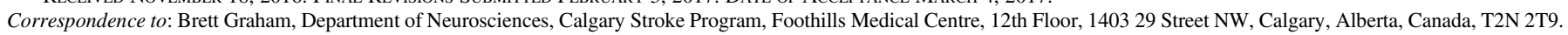
Email: brett.graham@ahs.ca 


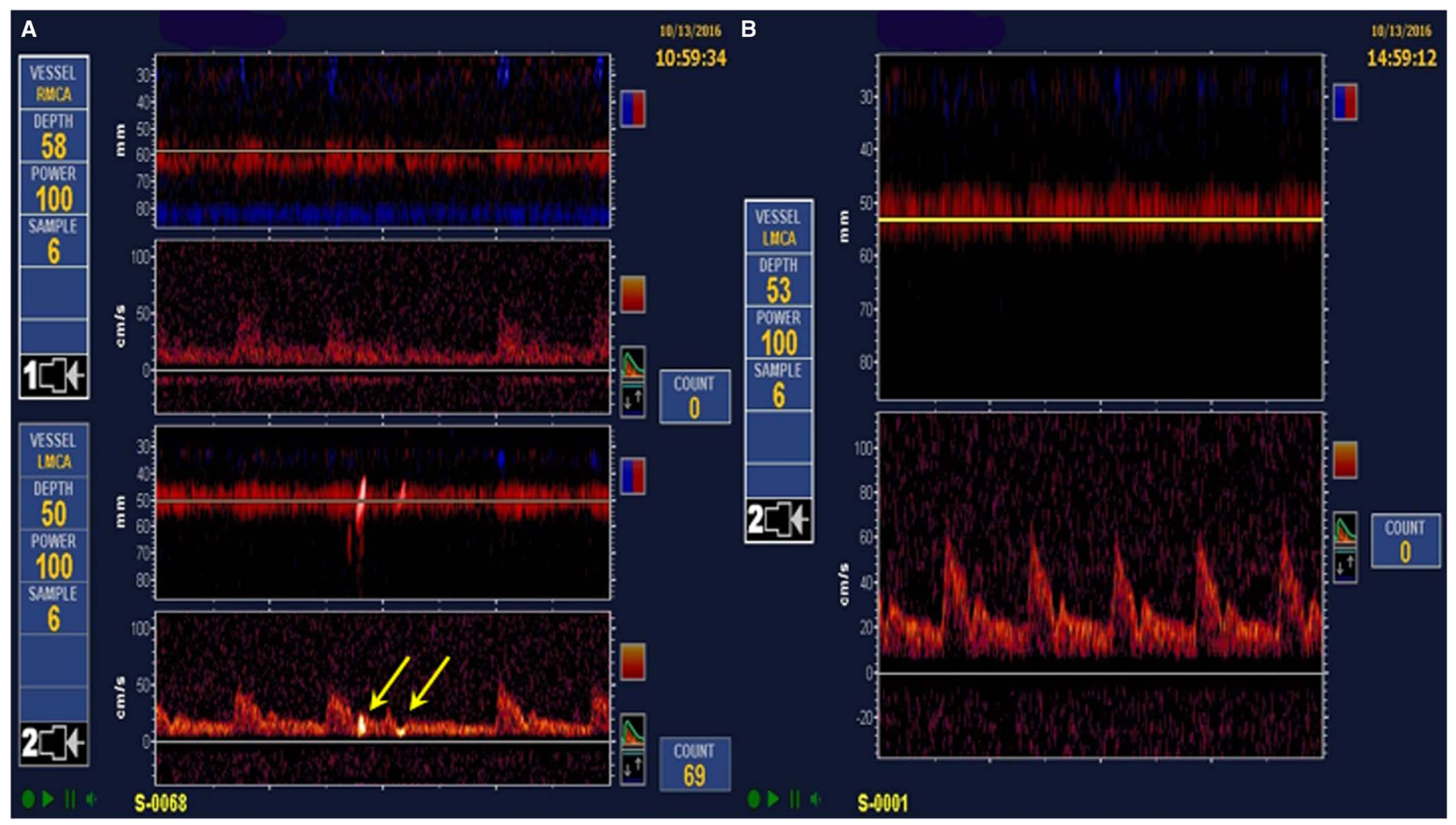

Figure 2: TCD studies before and after clopidogrel load. Multiple HITS, as depicted by yellow arrows, were observed in the left middle cerebral artery territory before loading (A). Four hours after clopidogrel load, no further HITS were detected (B).

TCD ultrasound is frequently used to detect microembolic signals (MES) in ischemic stroke, especially in the setting of extracranial large artery disease, ${ }^{1}$ although they have been recorded in other embolic etiologies as well. ${ }^{2}$ MES have been shown to be an independent risk factor for recurrent ischemic stroke in both symptomatic ${ }^{3}$ and asymptomatic carotid disease, ${ }^{4}$ and can be thought of as a surrogate marker of cerebral infarction. ${ }^{5}$

There are currently no guidelines regarding treatment of FFTs; as such, optimal treatment regimen is unclear. Interestingly, dual antiplatelet therapy has been shown to decrease the burden of MES in those with either extracranial ${ }^{6,7}$ or intracranial carotid disease, ${ }^{8}$ but not necessarily associated with FFTs. Furthermore, this decrease in embolization occurs over a period of days, not hours. The addition of a clopidogrel load to aspirin monotherapy has also been shown to decrease the number of asymptomatic MES in those with symptomatic extracranial carotid disease. ${ }^{9}$ A clopidogrel load of $600 \mathrm{mg}$ was chosen because it has been shown to have greater platelet activation with faster onset of action compared to 300-mg loads. ${ }^{10}$ Dual antiplatelet therapy has also been shown to predict decreased infarct burden on postcarotid stenting magnetic resonance imaging of the brain. ${ }^{11}$

Clopidogrel loading added to existing antithrombotic therapy may help decrease the burden of symptomatic and asymptomatic MES in a matter of hours, as suggested by this case given the temporal association between clopidogrel load and MES cessation. It further demonstrates the importance of using real-time monitoring of therapeutic interventions with TCDs. However, because treatment guidelines for FFTs are currently nonexistent, we would suggest caution in initiating triple antithrombotic therapy without careful consideration due to increased risk of bleeding.

\section{Disclosures}

The authors do not have anything to disclose.

\section{Statement of Authorship}

BRG and BKM were both involved in the care of the patient in this report. BRG prepared the manuscript and BKM proofread and revised it. Both authors read and approved the final manuscript.

\section{REFERENCES}

1. Babikian V, Hyde C, Pochy V, Winter M. Clinical correlates of high-intensity transient signals detected on transcranial Doppler sonography in patients with cerebrovascular disease. Stroke. 1994;25:1570-3.

2. Tong DC, Albers GW. Transcranial Doppler-detected microemboli in patients with acute stroke. Stroke. 1995;26:1588-92.

3. King A, Markus HS. Doppler embolic signals in cerebrovascular disease and prediction of stroke risk: a systematic review and meta-analysis. Stroke. 2009;40:3711-7.

4. Markus HS, King A, Shipley M, et al. Asymptomatic embolisation for prediction of stroke in the Asymptomatic Carotid Emboli Study (ACES): a prospective observational study. Lancet Neurol. 2010;9:663-71.

5. Wong KS. Is the measurement of cerebral microembolic signals a good surrogate marker for evaluating the efficacy of antiplatelet agents in the prevention of stroke? Eur Neurol. 2005;53:132-9. 
6. Markus HS, Droste DW, Kaps M, et al. Dual antiplatelet therapy with clopidogrel and aspirin in symptomatic carotid stenosis evaluated using Doppler embolic signal detection: the Clopidogrel and Aspirin for Reduction of Emboli in Symptomatic Carotid Stenosis (CARESS) trial. Circulation. 2005;111: 2233-2240.

7. Wong KSL, Chen $\mathrm{C}, \mathrm{Fu}$ J, et al. Clopidogrel plus aspirin versus aspirin alone for reducing embolisation in patients with acute symptomatic cerebral or carotid artery stenosis (CLAIR study): a randomised, open-label, blinded-endpoint trial. Lancet Neurol. 2010;9:489-97.

8. Wang X, Lin WH, Zhao YD, et al. The effectiveness of dual antiplatelet treatment in acute ischemic stroke patients with intracranial arterial stenosis: a subgroup analysis of CLAIR study. Int J Stroke. 2013;8:663-8.

9. Tsivgoulis G, Kerasnoudis A, Krogias C, et al. Clopidogrel load for emboli reduction in patients with symptomatic carotid stenosis undergoing urgent carotid endarterectomy. Stroke. 2012;43:1957-60.

10. Montalescot G, Sideris G, Meuleman C, Bal-dit-Sollier C, Lellouche N, Steg G. Clopidogrel loading doses in patients with nonST-segment elevation acute coronary syndromes. J Am Coll Cardio. 2006;48:931-8.

11. Almekhlafi MA, Demchuk AM, Mishra S, et al. Malignant emboli on transcranial Doppler during carotid stenting predict postprocedure diffusion-weighted imaging lesions. Stroke. 2013;44:1317-22. 